\title{
TRANSLATION INVARIANT SIGMA ALGEBRAS ON GROUPS
}

\author{
D. A. LIND
}

ABstract. The translation invariant sigma subalgebras of the Baire sets of a locally compact abelian group are characterized as those periodic under some closed subgroup.

One of the simplest examples of measure preserving transformations in ergodic theory is translation on a locally compact abelian group with Haar measure. When studying the ergodic properties of endomorphisms of such groups, it is of interest to know the sigma subalgebras of the measure algebra of Baire sets which are invariant under all translations. For compact abelian groups, Adler [1] impicitly and Rohlin [4] explicitly showed that the invariant sigma subalgebras are those classes of sets which are periodic under some closed subgroup. We give here a proof of this fact for locally compact abelian groups which uses only the StoneWeierstrass theorem and some approximate identity arguments.

Before going on, we need some notation and terminology. Let $G$ be a locally compact abelian group with Haar measure $m$. The class of Baire sets of $G$ is the sigma algebra generated by the compact $G_{\delta}$ sets. Let $\mathscr{B}(G)$ denote the measure algebra of Baire sets modulo null sets. The elements of $\mathscr{B}(G)$ are therefore equivalence classes $E$ of Baire sets $e \subset G$ on which the metric $\rho(E, F)=m(e \backslash f)+m(f \backslash e)$ is well defined. If $H$ is a closed subgroup of $G$, then $E \in \mathscr{B}(G)$ is called $H$-periodic if $\rho(E+h, E)=0$ for all $h \in H$. The sigma algebra of $H$-periodic sets, which we denote by $\mathscr{B}(G)_{H}$, is clearly invariant; that is, if $E \in \mathscr{B}(G)_{H}$ and $g \in G$, then $E+g \in \mathscr{B}(G)_{H}$. We will show that these exhaust the invariant sigma subalgebras of $\mathscr{B}(G)$.

In the case $G$ is the circle group $T$, the result reduces to the fact that the only invariant sigma subalgebras of $\mathscr{B}(T)$ are the $2 \pi / n$ periodic ones, where $n \geqq 1$. Another consequence is that the translates of any subset of the reals $R$ of finite positive measure generate $\mathscr{B}(R)$.

Since either a Baire set or its complement is sigma bounded, we will assume without loss that all Baire sets considered are sigma bounded. A sigma subalgebra of $\mathscr{B}(G)$ is called aperiodic if it is not contained in

Received by the editors February 5, 1973.

AMS (MOS) subject classifications (1970). Primary 28A65, 28A70; Secondary 28A05.

Key words and phrases. Translation invariant sigma algebras, closed subgroup, periodic Baire sets, Stone-Weierstrass theorem.

(c) American Mathematical Society 1974 
any $\mathscr{B}(G)_{H}$ for $H \neq 0$. If $X$ is a locally compact space, we let $\mathscr{B}(X)$ denote the class of Baire sets of $X$, and $C_{c}(X)$ denote the algebra of continuous functions on $X$ with compact support.

We will first prove that if a sigma subalgebra $\Sigma$ has no periodicity, then $\Sigma$ is all of $\mathscr{B}(G)$. For general $\Sigma$, we will divide out from $G$ the periodicity of $\Sigma$, project $\Sigma$ onto an aperiodic subalgebra of the Baire sets of the quotient, and apply the first result.

THEOREM 1. If $\Sigma$ is an aperiodic invariant sigma subalgebra of $\mathscr{B}(G)$, then $\Sigma=\mathscr{B}(G)$.

ProOf. First assume that $G$ is sigma compact, so that $G=\bigcup_{n=1}^{\infty} K_{n}$, where $K_{n}$ is a compact Baire set. Let $C_{\Sigma}(G)$ denote the algebra of bounded $\Sigma$-measurable continuous functions on $G$. We show that $C_{\Sigma}(G)$ is point separating. If $x \neq y$, aperiodicity of $\Sigma$ implies there is an $E \in \Sigma$ for which $\rho(E-x, E-y)>0$. It follows that there is a $g \in C_{c}(G)$ such that

$$
\int_{G} g(-t)\left[\chi_{E-x}(t)-\chi_{E-y}(t)\right] d m(t) \neq 0,
$$

where $\chi_{E}$ is the characteristic function of $E$. Then $g * \chi_{E} \in C_{\Sigma}(G)$, and $g * \chi_{E}(x) \neq g * \chi_{E}(y)$. This establishes that $C_{\Sigma}(G)$ separates points. Letting $C_{\Sigma}\left(K_{n}\right)$ denote the restriction of $C_{\Sigma}(G)$ to $K_{n}$, we see that $C_{\Sigma}\left(K_{n}\right)$ is a point separating, selfadjoint, uniformly closed subalgebra of $C\left(K_{n}\right)$. Hence $C_{\Sigma}\left(K_{n}\right)=C\left(K_{n}\right)$ by the Stone-Weierstrass theorem. Thus $\Sigma \cap K_{n} \equiv$ $\left\{E \cap K_{n}: E \in \Sigma\right\}$ contains all of the Baire subsets of $K_{n}$, which implies $\Sigma$ contains $\mathscr{B}(G)$, whence $\Sigma=\mathscr{B}(G)$.

Now consider a general group $G$. If $E \in \mathscr{B}(G)$, there is a sigma compact full Baire subgroup $Y$ of $G$ containing $E$ [2, Theorem 58B]. Since $Y$ is sigma compact and $\Sigma \cap Y$ is invariant and aperiodic in $\mathscr{B}(Y)$, the first paragraph implies $\Sigma \cap Y=\mathscr{B}(Y)$. Hence there is an $F \in \Sigma$ such that $F \cap Y=E$. Since $Y$ is Baire, we may assume that $F$ is sigma bounded, so that $F$ lies in a countable number of cosets of $Y$. Thus if $\pi_{Y}: G \rightarrow G / Y$ is the quotient map, there is a countable subgroup $D$ of the discrete group $G / Y$ such that $F \subset Y_{0} \equiv \pi_{\bar{Y}}^{-1}(D)$. Now $Y_{0}$ is a sigma compact group, and $\Sigma \cap Y_{0}$ is invariant and aperiodic, and so $\Sigma \cap Y_{0}=\mathscr{B}\left(Y_{0}\right)$. Hence there is an $F_{0} \in \Sigma$ with $F_{0} \cap Y_{0}=Y$. Thus $E=F \cap F_{0} \in \Sigma$. This shows $\Sigma=\mathscr{B}(G)$, and completes the proof.

If $H$ is a closed subgroup of $G$, then $G$ acts by translation on both $\mathscr{B}(G)_{H}$ and $\mathscr{B}(G / H)$. We need the following result in order to project $H$-periodic Baire sets into $\mathscr{B}(G / H)$.

LEMMA. Let $H$ be a closed subgroup of $G$. Then there is an injective sigma homomorphism $p: \mathscr{B}(G)_{H} \rightarrow \mathscr{B}(G / H)$ which commutes with the action of $G$. 
ProOF. This follows trivially from the existence of liftings of $L^{\infty}(G)$ which commute with translations [3]. Here is a more elementary proof.

Let $E \in \mathscr{B}(G)_{H}, m(E)>0$, and $e \in E$. As before, there is a sigma compact full subgroup $Y$ of $G$ containing $e$. Then there is a compact subgroup $Z$ of $Y$ such that $Y \mid Z$ is first countable and $E$ is $Z$-periodic [2, Theorem 64G]. This implies that there is a countable approximate identity $\psi_{n} \in C_{c}(Y \mid Z)$. Since $Z$ is compact, $\varphi_{n} \equiv \psi_{n} \pi_{Z}$ is in $C_{c}(Y)$, where $\pi_{Z}: Y \rightarrow Y \mid Z$. Now $\pi_{Z}(e)$ is Baire [2, Theorem 63F], so that $\psi_{n} * \chi_{\pi_{Z}(e)} \rightarrow \chi_{\pi_{Z}}(e)$ a.e. Compactness of $Z$ then gives $\varphi_{n} * \chi_{e} \rightarrow \chi_{e}$ a.e. on $Y$. Define $\bar{e}$ to be $\{y \in Y$ : $\left.\varphi_{n} * \chi_{e}(y) \rightarrow 1\right\}$. Then $\rho(e, \bar{e})=0, \bar{e}$ depends only on the equivalence class of $e$, and $\bar{e}+h=\bar{e}$ for all $h \in H$.

Note that $H \subset Y$. For if $h \in H, m[(Y+h) \cap Y] \geqq m[(e+h) \cap e]=m(e)>0$. If $\pi_{H}: Y \rightarrow Y / H$, we will show $\pi_{H}(\bar{e}) \in \mathscr{B}(Y / H) \subset \mathscr{B}(G / H)$. Now $\varphi_{n} * \chi_{e}$ is constant on the cosets of $H$ in $Y$. Thus there are functions $g_{n} \in C(Y \mid H)$ such that $g_{n} \pi_{H}=\varphi_{n} * \gamma_{e}$. It follows that $\pi_{H}(\bar{e})=\left\{y+H: g_{n}(y+H) \rightarrow 1\right\}$. The functions $g_{n}$ are continuous on the sigma compact set $Y$, and are therefore Baire measurable. Hence $\pi_{H}(\bar{e})$ is a Baire set. We define $p(E)$ to be the class of $\pi_{H}(\bar{e})$ in $\mathscr{B}(G / H)$. Then $p$ is a well-defined sigma homomorphism, $p^{-1} p(E)=E$ since $\rho(e, \bar{e})=0$ which shows that $p$ is injective, and $p$ clearly commutes with the action of $G$.

THEOREM 2. If $\Sigma$ is an invariant sigma subalgebra of $\mathscr{B}(G)$, then there is a closed subgroup $H$ of $G$ such that $\Sigma=\mathscr{B}(G)_{H}$.

Proof. Let $H=\{h \in G: \rho(E+h, E)=0$ for all $E \in \Sigma\}$. Clearly $H$ is a closed subgroup of $G$, and $\Sigma \subset \mathscr{B}(G)_{H}$. By the Lemma, there is a sigma homomorphism $p: \mathscr{B}(G)_{H} \rightarrow \mathscr{B}(G / H)$ commuting with translation. Therefore $p \Sigma$ is an aperiodic invariant subalgebra of $\mathscr{B}(G / H)$. By Theorem 1, $p \Sigma=\mathscr{B}(G / H)$, so that $\Sigma=p^{-1} p(\Sigma)=p^{-1} \mathscr{B}(G / H)=\mathscr{B}(G)_{H}$.

REMARKS. 1. If $G$ is assumed to be sigma compact in the Lemma, the proof shows that $p$ is a sigma isomorphism. However, in general $p$ need not be onto. For example, let $G$ be an uncountable discrete group and $H$ an uncountable proper subgroup. Since the Baire sets of $G$ are just the countable and cocountable sets, $\mathscr{B}(G)_{H}=\{\varnothing, G\}$. However, $\mathscr{B}(G / H)$ consists of the countable and cocountable unions of cosets of $H$.

2. The result here is still valid if $\mathscr{B}(G)$ is replaced by the sigma algebra generated by the compact sets (Halmos' Borel sets) since the resulting measure algebras are identical. However, it is false for the Borel sets as usually defined (the sigma algebra generated by the closed sets). For example, let $G$ be the discrete reals, and $\Sigma$ the sigma algebra generated by the sets which are of first category in the reals with the usual topology. Then $\Sigma$ is not a $\mathscr{B}(G)_{H}$ for any subgroup $H$ of $G$. 
3. Different closed subgroups may give rise to the same sigma subalgebra. As an example, in Remark 1 any uncountable subgroup of $G$ has $\{\varnothing, G\}$ as the induced invariant subalgebra.

4. The periodicity subgroup of an invariant subalgebra need not be a Baire set. If $G=\prod_{t \in R}\{0,1\}, H=\{g \in G: g(t)=0$ for $t \neq 0\}$, then $H$ is not Baire, while the periodicity subgroup of $\mathscr{B}(G)_{H}$ is $H$.

I thank Karel deLeeuw for several useful suggestions regarding this work.

\section{REFERENCES}

1. R. L. Adler, Invariant and reducing subalgebras of measure preserving transformations, Trans. Amer. Math. Soc. 110 (1964), 350-360. MR 28 \#183.

2. P. R. Halmos, Measure theory, Van Nostrand, Princeton, N.J., 1950. MR 11, 504.

3. A. Ionescu Tulcea and C. Ionescu Tulcea, On the existence of a lifting commuting with the left translations of an arbitrary locally compact group, Proc. Fifth Berkeley Sympos. Math. Statist. and Probability (Berkeley, Calif., 1965/66), Vol. II, Contributions to Probability Theory, part 1, Univ. of California Press, Berkeley, Calif., 1967, pp. 63-97. MR 35 \#2997.

4. V. A. Rohlin, A "general" measure-preserving transformation is not mixing, Dokl. Akad. Nauk SSSR 60 (1948), 349-351. (Russian) MR 9, 504.

Department of Mathematics, Stanford University, Stanford, California 94305

Current address: Department of Mathematics, University of California, Berkeley, California 94720 